\title{
DIRICHLET THEOREM FOR JACOBI-DUNKL EXPANSIONS
}

\author{
FREJ CHOUCHENE, INESS HAOUALA \\ DEPARTMENT OF MATHEMATICS, \\ HIGHER SCHOOL OF SCIENCES AND TECHNOLOGY OF HAMMAM SOUSSE, \\ UNIVERSITY OF SOUSSE, TUNISIA.
}

\begin{abstract}
The purpose of this paper is to study the pointwise convergence of the Jacobi-Dunkl series. Indeed, we recall some properties of the Jacobi-Dunkl coefficients. Then, we establish a Dirichlet type theorem for expansions in term of Jacobi-Dunkl polynomials.
\end{abstract}

\section{InTRODUCTION}

The Fourier series is named in honour of Jean-Baptiste Joseph Fourier (1768-1830) who introduced it for the purpose of solving the heat equation in a metal plate, publishing his initial results in [6], and pursuing his study in [7]. The question whether the Fourier series of a periodic function converges to a given function is well researched and an extensive literature exists on this subject. We mention here for example $[9,5,12]$. Indeed, mathematicians studied pointwise, absolute, uniform, quadratic convergences... It is well known that many problems for partial differential equations are reduced to a power series expansion of the desired solution in terms of special functions or orthogonal polynomials. In particular, by using the properties of Jacobi polynomials ([15]), the Fourier-Jacobi series has been studied extensively by many authors and several results concerning the approximation of functions by partial sums of these series are proved (see e.g. [1, 11, 13, 14, 16, 17, 18]).

In this paper, we also discuss this subject. More precisely, we are interested in JacobiDunkl expansions.

In [2], the author defined the Jacobi-Dunkl coefficients associated with Jacobi-Dunkl polynomials given by

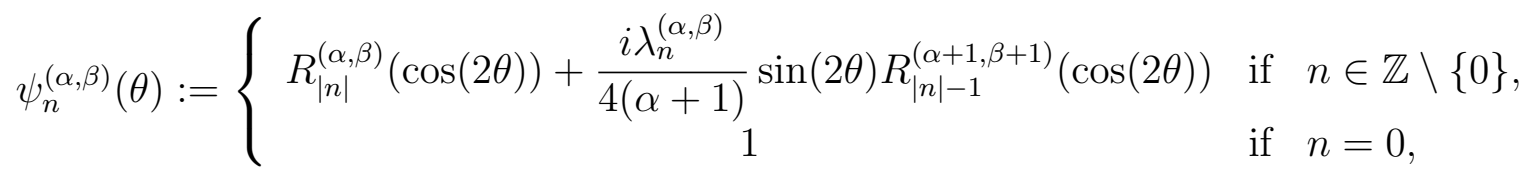

2010 Mathematics Subject Classification. 33B10, 33C45, 33C47, 40A30, 42A10, 42A16.

Key words and phrases. Jacobi-Dunkl polynomials, Jacobi-Dunkl coefficients, Jacobi-Dunkl series, quadratic mean convergence, Dirichlet type theorem. 
where $R_{m}^{(\alpha, \beta)}(x), m \in \mathbb{N}$, is the normalized Jacobi polynomial of degree $m$ such that $R_{m}^{(\alpha, \beta)}(1)=1$, and $\lambda_{n}^{(\alpha, \beta)}$ is given by

$$
\lambda_{n}^{(\alpha, \beta)}:=2 \operatorname{sgn}(n) \sqrt{|n|(|n|+\rho)}, \quad n \in \mathbb{Z},
$$

with

$$
\alpha \geq \beta \geq-\frac{1}{2} ; \quad \alpha \neq-\frac{1}{2}, \text { and } \rho:=\alpha+\beta+1>0
$$

In the second section, we will give some preliminaries concerning these polynomials. Then, we will see more properties of the Jacobi-Dunkl coefficients in the third section. In section 4, we state a theorem about Jacobi-Dunkl convergence in quadratic mean. Finally, we will focus on pointwise convergence. We establish a Dirichlet type theorem which generalizes the classical one, see [10]. The proof is based on the asymptotic behaviour of Jacobi and Jacobi-Dunkl polynomials studied in [3] and [4].

\section{Preliminaries}

In this section, we will recall some properties of Jacobi and Jacobi-Dunkl polynomials. We denote by

$$
(a)_{n}:=\left\{\begin{array}{cl}
a(a+1) \ldots(a+n-1) & \text { if } n \in \mathbb{N} \backslash\{0\} \\
1 & \text { if } n=0 .
\end{array}\right.
$$

$(a)_{n}$ is called the Pochhammer symbol.

${ }_{2} F_{1}(a, b ; c ; z)$ is the Gauss hypergeometric function, given by

$$
\forall a, b \in \mathbb{C}, \forall c \in \mathbb{C} \backslash \mathbb{Z}_{-}, \forall z \in \mathbb{C} ;|z|<1, \quad{ }_{2} F_{1}(a, b ; c ; z):=\sum_{n=0}^{+\infty} \frac{(a)_{n}(b)_{n}}{n !(c)_{n}} z^{n} .
$$

The Jacobi polynomials $\varphi_{m}^{(\alpha, \beta)}(\theta), m \in \mathbb{N}, \theta \in\left[-\frac{\pi}{2}, \frac{\pi}{2}\right]$, are defined by

$$
\varphi_{m}^{(\alpha, \beta)}(\theta):=R_{m}^{(\alpha, \beta)}(\cos (2 \theta))={ }_{2} F_{1}\left(-m, m+\rho ; \alpha+1 ;(\sin \theta)^{2}\right) .
$$

The Jacobi operator $\Delta_{\alpha, \beta}$ defined on $C^{2}(] 0, \frac{\pi}{2}[)$ is given by

$$
\Delta_{\alpha, \beta}:=\frac{d^{2}}{d \theta^{2}}+\frac{A_{\alpha, \beta}^{\prime}}{A_{\alpha, \beta}} \frac{d}{d \theta},
$$

where

$$
A_{\alpha, \beta}(\theta):=\left\{\begin{array}{cl}
2^{2 \rho}(\sin |\theta|)^{2 \alpha+1}(\cos \theta)^{2 \beta+1} & \text { if } \theta \in]-\frac{\pi}{2}, \frac{\pi}{2}[\backslash\{0\} \\
0 & \text { if } \theta=0 .
\end{array}\right.
$$

For all $m \in \mathbb{N}, \varphi_{m}^{(\alpha, \beta)}$ is the unique even $\mathcal{C}^{\infty}$-solution on $]-\frac{\pi}{2}, \frac{\pi}{2}[$ of the differential equation

$$
\left\{\begin{aligned}
\Delta_{\alpha, \beta} u & =-\lambda_{m}^{2} u \\
u(0) & =1 \\
u^{\prime}(0) & =0
\end{aligned}\right.
$$


The Jacobi-Dunkl operator $\wedge_{\alpha, \beta}$ is defined by

$$
\wedge_{\alpha, \beta} f(\theta):=\frac{d}{d \theta} f(\theta)+\frac{A_{\alpha, \beta}^{\prime}(\theta)}{A_{\alpha, \beta}(\theta)} \frac{f(\theta)-f(-\theta)}{2}, \quad f \in C^{1}(]-\frac{\pi}{2}, \frac{\pi}{2}[),
$$

with $\left.\frac{A_{\alpha, \beta}^{\prime}(\theta)}{A_{\alpha, \beta}(\theta)}=(2 \alpha+1) \cot \theta-(2 \beta+1) \tan \theta, \quad \theta \in\right]-\frac{\pi}{2}, \frac{\pi}{2}[\backslash\{0\}$.

According to $[2]$, the differential-difference equation

$$
\left\{\begin{array}{cl}
\wedge_{\alpha, \beta} u(\theta) & =i \lambda_{n}^{(\alpha, \beta)} u(\theta) ; \quad n \in \mathbb{Z} \\
u(0) & =1
\end{array}\right.
$$

admits a unique $\mathcal{C}^{\infty}$-solution on $]-\frac{\pi}{2}, \frac{\pi}{2}[$ given by (1), which is related to the Jacobi polynomial and to its derivative by

$$
\psi_{n}^{(\alpha, \beta)}(\theta):=\left\{\begin{array}{clc}
\varphi_{|n|}^{(\alpha, \beta)}(\theta)-\frac{i}{\lambda_{n}^{(\alpha, \beta)}} \frac{d}{d \theta} \varphi_{|n|}^{(\alpha, \beta)}(\theta) & \text { if } & n \in \mathbb{Z} \backslash\{0\} \\
1 & \text { if } \quad n=0
\end{array}\right.
$$

and satisfies

$$
\forall n \in \mathbb{Z}, \forall \theta \in\left[-\frac{\pi}{2}, \frac{\pi}{2}\right], \quad\left|\psi_{n}^{(\alpha, \beta)}(\theta)\right| \leq 1 .
$$

For all $n, p \in \mathbb{Z}$, we have the following orthogonality property

$$
\int_{-\frac{\pi}{2}}^{\frac{\pi}{2}} \psi_{n}^{(\alpha, \beta)}(\theta) \overline{\psi_{p}^{(\alpha, \beta)}(\theta)} A_{\alpha, \beta}(\theta) d \theta=\left(h_{n}^{(\alpha, \beta)}\right)^{-1} \delta_{n, p},
$$

where $h_{n}^{(\alpha, \beta)}=\left(\int_{-\frac{\pi}{2}}^{\frac{\pi}{2}}\left|\psi_{n}^{(\alpha, \beta)}(\theta)\right|^{2} A_{\alpha, \beta}(\theta) d \theta\right)^{-1}: h_{0}^{(\alpha, \beta)}=\frac{\Gamma(\rho+1)}{2^{2 \rho} \Gamma(\alpha+1) \Gamma(\beta+1)}$

and

$$
\forall n \in \mathbb{Z} \backslash\{0\}, h_{n}^{(\alpha, \beta)}=\frac{(2|n|+\rho) \Gamma(\alpha+|n|+1) \Gamma(\rho+|n|)}{2^{2 \rho+1}(\Gamma(\alpha+1))^{2} \Gamma(|n|+1) \Gamma(\beta+|n|+1)} .
$$

Let $p \in[1,+\infty]$. We denote by

- $L_{\alpha, \beta}^{p}=L^{p}\left(\left[-\frac{\pi}{2}, \frac{\pi}{2}\right], A_{\alpha, \beta}(\theta) d \theta\right)$ : the space of measurable functions $f$ on $\left[-\frac{\pi}{2}, \frac{\pi}{2}\right]$ such that

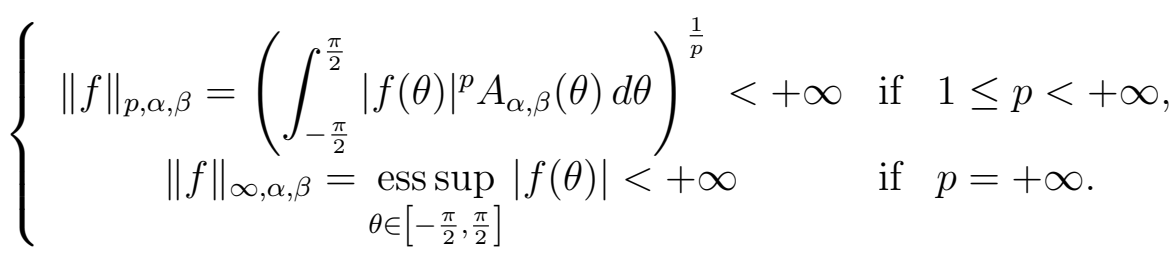

- $\widetilde{L}_{\alpha, \beta}^{p}=L^{p}\left(\left[0, \frac{\pi}{2}\right], A_{\alpha, \beta}(\theta) d \theta\right)$ the space of measurable functions $g$ on $\left[0, \frac{\pi}{2}\right]$ such that

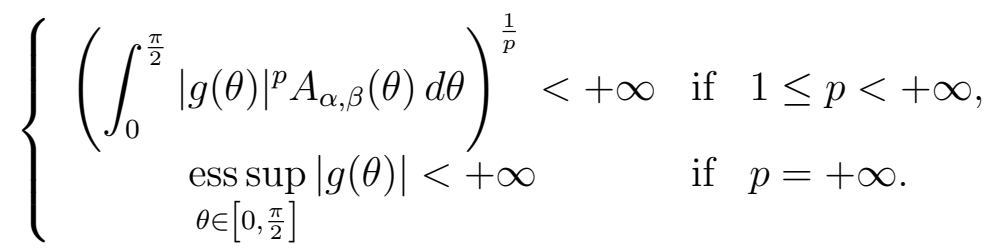


The Jacobi coefficients (see [8]) of a function $g \in \widetilde{L}_{\alpha, \beta}^{1}$ are defined by

$$
\forall m \in \mathbb{N}, \quad \mathcal{F}_{\alpha, \beta}(g)(m)=\int_{0}^{\frac{\pi}{2}} g(\theta) \varphi_{m}^{(\alpha, \beta)}(\theta) A_{\alpha, \beta}(\theta) d \theta .
$$

The Jacobi-Dunkl coefficients (see [2]) of a function $f \in L_{\alpha, \beta}^{1}$ are defined by

$$
\forall n \in \mathbb{Z}, \quad \mathcal{F} f(n):=\int_{-\frac{\pi}{2}}^{\frac{\pi}{2}} f(\theta) \overline{\psi_{n}^{(\alpha, \beta)}(\theta)} A_{\alpha, \beta}(\theta) d \theta,
$$

and satisfy

$$
\forall n \in \mathbb{Z}, \quad|\mathcal{F} f(n)| \leq\|f\|_{1, \alpha, \beta} .
$$

Now, we consider the analog of the Fourier series given by

$$
\sum_{n=-\infty}^{+\infty} \mathcal{F} f(n) \psi_{n}^{(\alpha, \beta)}(\theta) h_{n}^{(\alpha, \beta)}, \quad \theta \in\left[-\frac{\pi}{2}, \frac{\pi}{2}\right] .
$$

For $n \in \mathbb{N}$, we denote its partial sum by

$$
S_{n}^{f}(\theta):=\sum_{k=-n}^{n} \mathcal{F} f(k) \psi_{k}^{(\alpha, \beta)}(\theta) h_{k}^{(\alpha, \beta)}, \quad \theta \in\left[-\frac{\pi}{2}, \frac{\pi}{2}\right] .
$$

\section{JACOBI-DUNKL COEFFICIENTS}

Let $f \in L_{\alpha, \beta}^{1}$. We put for all $k \in \mathbb{N}$,

$$
a_{k}(f):=\mathcal{F} f(k)+\mathcal{F} f(-k),
$$

and

$$
b_{k}(f):=\left\{\begin{array}{cll}
-\frac{i}{\lambda_{k}^{(\alpha, \beta)}}[\mathcal{F} f(k)-\mathcal{F} f(-k)] & \text { if } & k \in \mathbb{N} \backslash\{0\}, \\
0 & \text { if } \quad k=0 .
\end{array}\right.
$$

Hence, by (2) we can write $S_{n}^{f}(\theta)$, for $n \in \mathbb{N} \backslash\{0\}$ and $\theta \in\left[-\frac{\pi}{2}, \frac{\pi}{2}\right]$, as

$$
S_{n}^{f}(\theta)=\frac{a_{0}(f)}{2} h_{0}^{(\alpha, \beta)}+\sum_{k=1}^{n}\left(a_{k}(f) \varphi_{k}^{(\alpha, \beta)}(\theta)+b_{k}(f) \frac{d}{d \theta} \varphi_{k}^{(\alpha, \beta)}(\theta)\right) h_{k}^{(\alpha, \beta)} .
$$

\section{Remark 3.1.}

For all $k \in \mathbb{N}$, we have these relations:

(1) $\mathcal{F} f(k)=\frac{a_{k}(f)+i \lambda_{k}^{(\alpha, \beta)} b_{k}(f)}{2}$.

(2) $\mathcal{F} f(-k)=\frac{a_{k}(f)-i \lambda_{k}^{(\alpha, \beta)} b_{k}(f)}{2}$.

\section{Proposition 3.2.}

For all $k \in \mathbb{N}$, we have the following integral representations:

(1) $a_{k}(f)=2 \int_{-\frac{\pi}{2}}^{\frac{\pi}{2}} f(\theta) \varphi_{k}^{(\alpha, \beta)}(\theta) A_{\alpha, \beta}(\theta) d \theta$. 
(2) $b_{k}(f)=\frac{2}{\left(\lambda_{k}^{(\alpha, \beta)}\right)^{2}} \int_{-\frac{\pi}{2}}^{\frac{\pi}{2}} f(\theta) \frac{d}{d \theta} \varphi_{k}^{(\alpha, \beta)}(\theta) A_{\alpha, \beta}(\theta) d \theta, \quad k \neq 0$.

Proof.

(1) $a_{k}(f)=\mathcal{F} f(k)+\mathcal{F} f(-k)=\int_{-\frac{\pi}{2}}^{\frac{\pi}{2}} f(\theta)\left[\overline{\psi_{k}^{(\alpha, \beta)}(\theta)}+\overline{\psi_{-k}^{(\alpha, \beta)}(\theta)}\right] A_{\alpha, \beta}(\theta) d \theta$.

Since we know that

$$
\overline{\psi_{k}^{(\alpha, \beta)}(\theta)}+\overline{\psi_{-k}^{(\alpha, \beta)}(\theta)}=2 \Re\left(\psi_{k}^{(\alpha, \beta)}(\theta)\right)=2 \varphi_{k}^{(\alpha, \beta)}(\theta),
$$

then, we obtain the result.

(2) $b_{k}(f)=\frac{i}{\lambda_{k}^{(\alpha, \beta)}} \int_{-\frac{\pi}{2}}^{\frac{\pi}{2}} f(\theta)\left[\overline{\psi_{-k}^{(\alpha, \beta)}(\theta)}-\overline{\psi_{k}^{(\alpha, \beta)}(\theta)}\right] A_{\alpha, \beta}(\theta) d \theta, \quad k \neq 0$.

As we have

$$
\overline{\psi_{-k}^{(\alpha, \beta)}(\theta)}-\overline{\psi_{k}^{(\alpha, \beta)}(\theta)}=2 i \Im\left(\psi_{k}^{(\alpha, \beta)}(\theta)\right)=-\frac{2 i}{\lambda_{k}^{(\alpha, \beta)}} \frac{d}{d \theta} \varphi_{|k|}^{(\alpha, \beta)}(\theta),
$$

then, we get the equality.

\section{Remarks 3.3.}

Let $k \in \mathbb{N}$.

(1) If the function $f$ is even, then

$$
b_{k}(f)=0 \quad \text { and } \quad a_{k}(f)=4 \int_{0}^{\frac{\pi}{2}} f(\theta) \varphi_{k}^{(\alpha, \beta)}(\theta) A_{\alpha, \beta}(\theta) d \theta .
$$

(2) If the function $f$ is odd, then

$$
a_{k}(f)=0 \quad \text { and } \quad b_{k}(f)=\frac{4}{\left(\lambda_{k}^{(\alpha, \beta)}\right)^{2}} \int_{0}^{\frac{\pi}{2}} f(\theta) \frac{d}{d \theta} \varphi_{k}^{(\alpha, \beta)}(\theta) A_{\alpha, \beta}(\theta) d \theta, \quad k \neq 0 .
$$

\section{Proposition 3.4.}

Let $f$ be in $L_{\alpha, \beta}^{1}$, a real-valued function. For all $k \in \mathbb{N}$, we have these properties:

(1) $\mathcal{F} f(-k)=\overline{\mathcal{F} f(k)}$.

(2) $a_{k}(f)=2 \Re(\mathcal{F} f(k)) \in \mathbb{R}$.

(3) $b_{k}(f)=\frac{2}{\lambda_{k}^{(\alpha, \beta)}} \Im(\mathcal{F} f(k)) \in \mathbb{R}, \quad k \neq 0$.

Proof.

(1) $\mathcal{F} f(-k)=\int_{-\frac{\pi}{2}}^{\frac{\pi}{2}} f(\theta) \overline{\psi_{-k}^{(\alpha, \beta)}(\theta)} A_{\alpha, \beta}(\theta) d \theta=\overline{\int_{-\frac{\pi}{2}}^{\frac{\pi}{2}} f(\theta) \overline{\psi_{k}^{(\alpha, \beta)}(\theta)} A_{\alpha, \beta}(\theta) d \theta}=\overline{\mathcal{F} f(k)}$. 
$(2) a_{k}(f)=\mathcal{F} f(k)+\mathcal{F} f(-k)=\mathcal{F} f(k)+\overline{\mathcal{F} f(k)}=2 \Re(\mathcal{F} f(k))$.

(3) For all $k \in \mathbb{Z} \backslash\{0\}$, we have

$$
b_{k}(f)=\frac{i}{\lambda_{k}^{(\alpha, \beta)}}[\mathcal{F} f(-k)-\mathcal{F} f(k)]=\frac{i}{\lambda_{k}^{(\alpha, \beta)}}[\overline{\mathcal{F} f(k)}-\mathcal{F} f(k)]=\frac{2}{\lambda_{k}^{(\alpha, \beta)}} \Im(\mathcal{F} f(k)) .
$$

In the following parts, we will study for a suitable given function $f$, the convergence of the series

$$
\sum_{n=-\infty}^{+\infty} \mathcal{F} f(n) \psi_{n}^{(\alpha, \beta)}(\theta) h_{n}^{(\alpha, \beta)}
$$

\section{Convergence in quadratic mean}

Theorem 4.1. For all $f \in L_{\alpha, \beta}^{2}$, we have

$$
\lim _{n \rightarrow+\infty}\left\|S_{n}^{f}-f\right\|_{2, \alpha, \beta}=0 .
$$

Proof. Let $f \in L_{\alpha, \beta}^{2}$ and $n \in \mathbb{N}$.

$$
\begin{aligned}
\int_{-\frac{\pi}{2}}^{\frac{\pi}{2}}\left|S_{n}^{f}(\theta)-f(\theta)\right|^{2} A_{\alpha, \beta}(\theta) d \theta & =\int_{-\frac{\pi}{2}}^{\frac{\pi}{2}}\left(S_{n}^{f}(\theta)-f(\theta)\right) \overline{\left(S_{n}^{f}(\theta)-f(\theta)\right)} A_{\alpha, \beta}(\theta) d \theta \\
& =\int_{-\frac{\pi}{2}}^{\frac{\pi}{2}}\left|S_{n}^{f}(\theta)\right|^{2} A_{\alpha, \beta}(\theta) d \theta-\int_{-\frac{\pi}{2}}^{\frac{\pi}{2}} S_{n}^{f}(\theta) \overline{f(\theta)} A_{\alpha, \beta}(\theta) d \theta \\
& -\int_{-\frac{\pi}{2}}^{\frac{\pi}{2}} f(\theta) \overline{S_{n}^{f}(\theta)} A_{\alpha, \beta}(\theta) d \theta+\int_{-\frac{\pi}{2}}^{\frac{\pi}{2}}|f(\theta)|^{2} A_{\alpha, \beta}(\theta) d \theta \\
& :=I_{1}+I_{2}+I_{3}+I_{4} .
\end{aligned}
$$

We have by the orthogonality property (3),

$$
\begin{aligned}
& I_{1}=\int_{-\frac{\pi}{2}}^{\frac{\pi}{2}}\left(\sum_{k=-n}^{n} \mathcal{F} f(k) \psi_{k}^{(\alpha, \beta)}(\theta) h_{k}^{(\alpha, \beta)}(\theta)\right) \overline{\left(\sum_{p=-n}^{n} \mathcal{F} f(p) \psi_{p}^{(\alpha, \beta)}(\theta) h_{p}^{(\alpha, \beta)}(\theta)\right)} A_{\alpha, \beta}(\theta) d \theta \\
& =\sum_{k=-n}^{n} \sum_{p=-n}^{n}\left(\mathcal{F} f(k) \overline{\mathcal{F} f(p)} h_{p}^{(\alpha, \beta)} h_{k}^{(\alpha, \beta)}\right)\left(\int_{-\frac{\pi}{2}}^{\frac{\pi}{2}} \psi_{k}^{(\alpha, \beta)}(\theta) \overline{\psi_{p}^{(\alpha, \beta)}(\theta)} A_{\alpha, \beta}(\theta) d \theta\right) \\
& =\sum_{k=-n}^{n} \sum_{p=-n}^{n} \mathcal{F} f(k) \overline{\mathcal{F} f(p)} h_{p}^{(\alpha, \beta)} h_{k}^{(\alpha, \beta)}\left(h_{k}^{(\alpha, \beta)}\right)^{-1} \delta_{k, p} \\
& =\sum_{k=-n}^{n} \mathcal{F} f(k) \overline{\mathcal{F} f(k)} h_{k}^{(\alpha, \beta)} \\
& =\sum_{k=-n}^{n}|\mathcal{F} f(k)|^{2} h_{k}^{(\alpha, \beta)} \text {. }
\end{aligned}
$$


Furthermore

$$
\begin{aligned}
I_{2} & =-\int_{-\frac{\pi}{2}}^{\frac{\pi}{2}}\left(\sum_{k=-n}^{n} \mathcal{F} f(k) \psi_{k}^{(\alpha, \beta)}(\theta) \overline{\left.h_{k}^{(\alpha, \beta)}\right) \overline{f(\theta)}} A_{\alpha, \beta}(\theta) d \theta\right. \\
& =-\sum_{k=-n}^{n} \mathcal{F} f(k) h_{k}^{(\alpha, \beta)} \overline{\left(\int_{-\frac{\pi}{2}}^{\frac{\pi}{2}} f(\theta) \overline{\psi_{k}^{(\alpha, \beta)}(\theta)} A_{\alpha, \beta}(\theta) d \theta\right)} \\
& =-\sum_{k=-n}^{n} \mathcal{F} f(k) \overline{\mathcal{F} f(k)} h_{k}^{(\alpha, \beta)} \\
& =-\sum_{k=-n}^{n}|\mathcal{F} f(k)|^{2} h_{k}^{(\alpha, \beta)} \\
& =-I_{1} .
\end{aligned}
$$

We also have

$$
I_{3}=\overline{I_{2}}=I_{2}=-I_{1}
$$

Then

$$
\int_{-\frac{\pi}{2}}^{\frac{\pi}{2}}\left|S_{n}^{f}(\theta)-f(\theta)\right|^{2} A_{\alpha, \beta}(\theta) d \theta=\|f\|_{2, \alpha, \beta}-\sum_{k=-n}^{n}|\mathcal{F} f(k)|^{2} h_{k}^{(\alpha, \beta)} .
$$

By the Plancherel formula [2, Theorem 3.4], we obtain

$$
\lim _{n \rightarrow+\infty} \int_{-\frac{\pi}{2}}^{\frac{\pi}{2}}\left|S_{n}^{f}(\theta)-f(\theta)\right|^{2} A_{\alpha, \beta}(\theta) d \theta=0 .
$$

\section{Dirichlet type CONVERGEnCE}

Notation 5.1.

For all $n \in \mathbb{N}, \theta, \phi \in\left[-\frac{\pi}{2}, \frac{\pi}{2}\right]$. We denote by

$$
D_{n}^{(\alpha, \beta)}(\theta, \phi):=\sum_{k=-n}^{n} \psi_{k}^{(\alpha, \beta)}(\theta) \overline{\psi_{k}^{(\alpha, \beta)}(\phi)} h_{k}^{(\alpha, \beta)}
$$

$D_{n}^{(\alpha, \beta)}(\theta, \phi)$ is the analog of the Dirichlet kernel associated with the Fourier series.

\section{Proposition 5.2.}

Let $f \in L_{\alpha, \beta}^{1}, n \in \mathbb{N}$ and $\theta \in\left[-\frac{\pi}{2}, \frac{\pi}{2}\right]$. We have

$$
S_{n}^{f}(\theta)=\int_{-\frac{\pi}{2}}^{\frac{\pi}{2}} f(\phi) D_{n}^{(\alpha, \beta)}(\theta, \phi) A_{\alpha, \beta}(\phi) d \phi
$$


Proof.

$$
\begin{aligned}
S_{n}^{f}(\theta) & =\sum_{k=-n}^{n}\left(\int_{-\frac{\pi}{2}}^{\frac{\pi}{2}} f(\phi) \overline{\psi_{k}^{(\alpha, \beta)}(\phi)} A_{\alpha, \beta}(\phi) d \phi\right) \psi_{k}^{(\alpha, \beta)}(\theta) h_{k}^{(\alpha, \beta)} \\
& =\int_{-\frac{\pi}{2}}^{\frac{\pi}{2}} f(\phi)\left(\sum_{k=-n}^{n} \overline{\psi_{k}^{(\alpha, \beta)}(\phi)} \psi_{k}^{(\alpha, \beta)}(\theta) h_{k}^{(\alpha, \beta)}\right) A_{\alpha, \beta}(\phi) d \phi \\
& =\int_{-\frac{\pi}{2}}^{\frac{\pi}{2}} f(\phi) D_{n}^{(\alpha, \beta)}(\theta, \phi) A_{\alpha, \beta}(\phi) d \phi .
\end{aligned}
$$

\section{Proposition 5.3.}

Let $n \in \mathbb{N}$ and $\theta \in\left[-\frac{\pi}{2}, \frac{\pi}{2}\right]$. We have

$$
\int_{-\frac{\pi}{2}}^{\frac{\pi}{2}} D_{n}^{(\alpha, \beta)}(\theta, \phi) A_{\alpha, \beta}(\phi) d \phi=1
$$

Proof.

$$
\int_{-\frac{\pi}{2}}^{\frac{\pi}{2}} D_{n}^{(\alpha, \beta)}(\theta, \phi) A_{\alpha, \beta}(\phi) d \phi=\sum_{k=-n}^{n}\left(\int_{-\frac{\pi}{2}}^{\frac{\pi}{2}} \overline{\psi_{k}^{(\alpha, \beta)}(\phi)} A_{\alpha, \beta}(\phi) d \phi\right) \psi_{k}^{(\alpha, \beta)}(\theta) h_{k}^{(\alpha, \beta)} .
$$

As we know, by the orthogonality property (3), that

$$
\int_{-\frac{\pi}{2}}^{\frac{\pi}{2}} \overline{\psi_{k}^{(\alpha, \beta)}(\phi)} A_{\alpha, \beta}(\phi) d \phi=\left(h_{0}^{(\alpha, \beta)}\right)^{-1} \delta_{0, k}
$$

then, we get

$$
\int_{-\frac{\pi}{2}}^{\frac{\pi}{2}} D_{n}^{(\alpha, \beta)}(\theta, \phi) A_{\alpha, \beta}(\phi) d \phi=\psi_{0}^{(\alpha, \beta)}(\theta)=1
$$

\section{Proposition 5.4.}

(1) $\forall n \in \mathbb{N}, \forall \theta, \phi \in\left[-\frac{\pi}{2}, \frac{\pi}{2}\right] ; \theta \neq \pm \phi$, we have

$$
\begin{aligned}
D_{n}^{(\alpha, \beta)}(\theta, \phi) & =\frac{\Gamma(\alpha+n+2) \Gamma(\rho+n+1)}{2^{2 \rho-1}(\Gamma(\alpha+1))^{2}(2 n+\rho+1) n ! \Gamma(\beta+n+1)} \times \frac{1}{\cos (2 \theta)-\cos (2 \phi)} \\
& \times\left[\varphi_{n+1}^{(\alpha, \beta)}(\theta) \varphi_{n}^{(\alpha, \beta)}(\phi)-\varphi_{n}^{(\alpha, \beta)}(\theta) \varphi_{n+1}^{(\alpha, \beta)}(\phi)+\frac{\lambda_{n}^{(\alpha, \beta)} \lambda_{n+1}^{(\alpha, \beta)}}{4(n+1)(n+\rho)}\right. \\
& \left.\times\left(\Im \psi_{n+1}^{(\alpha, \beta)}(\theta) \Im \psi_{n}^{(\alpha, \beta)}(\phi)-\Im \psi_{n}^{(\alpha, \beta)}(\theta) \Im \psi_{n+1}^{(\alpha, \beta)}(\phi)\right)\right],
\end{aligned}
$$

with $\Im \psi_{n}^{(\alpha, \beta)}(\theta)=\frac{\psi_{n}(\theta)-\psi_{n}(-\theta)}{2 i}$.

(2) $\forall n \in \mathbb{N}, \forall \theta, \phi \in\left[-\frac{\pi}{2}, \frac{\pi}{2}\right]$, we have

(a) $D_{n}^{(\alpha, \beta)}(\theta, \phi) \in \mathbb{R}$.

(b) $D_{n}^{(\alpha, \beta)}(\theta, \theta)>0$. 
(c) $D_{n}^{(\alpha, \beta)}(\phi, \theta)=D_{n}^{(\alpha, \beta)}(\theta, \phi)$.

Proof.

(1) The case $n=0$ is obvious, and we have the result in [4, theorem 3.1], for $n \in \mathbb{N} \backslash\{0\}$.

(2) (a) We deduce the result from (1), for $\theta \neq \pm \phi$. We also have

$$
D_{n}^{(\alpha, \beta)}(\theta, \theta)=\sum_{k=-n}^{n}\left|\psi_{k}^{(\alpha, \beta)}(\theta)\right|^{2} h_{k}^{(\alpha, \beta)} \in \mathbb{R}
$$

and

$$
\begin{aligned}
D_{n}^{(\alpha, \beta)}(\theta,-\theta) & =\sum_{k=-n}^{n}\left(\psi_{k}^{(\alpha, \beta)}(\theta)\right)^{2} h_{k}^{(\alpha, \beta)} \\
& =h_{0}^{(\alpha, \beta)}+\sum_{k=1}^{n}\left(\left(\psi_{k}^{(\alpha, \beta)}(\theta)\right)^{2}+\left(\overline{\left(\psi_{k}^{(\alpha, \beta)}(\theta)\right.}\right)^{2}\right) h_{k}^{(\alpha, \beta)} \\
& =h_{0}^{(\alpha, \beta)}+2 \Re\left(\sum_{k=1}^{n}\left(\psi_{k}^{(\alpha, \beta)}(\theta)\right)^{2}\right) \in \mathbb{R} .
\end{aligned}
$$

(b) $D_{n}^{(\alpha, \beta)}(\theta, \theta)=h_{0}^{(\alpha, \beta)}+\sum_{k=-n, k \neq 0}^{n}\left|\psi_{k}^{(\alpha, \beta)}(\theta)\right|^{2} h_{k}^{(\alpha, \beta)}>0$.

(c) $D_{n}^{(\alpha, \beta)}(\phi, \theta)=\overline{D_{n}^{(\alpha, \beta)}(\theta, \phi)}=D_{n}^{(\alpha, \beta)}(\theta, \phi)$.

Theorem 5.5.

Let $f$ be a piecewise continuous function on $\left[-\frac{\pi}{2}, \frac{\pi}{2}\right]$ and $\theta \in\left[-\frac{\pi}{2}, \frac{\pi}{2}\right] \backslash\{0\}$ such that

i) $f(-\theta)=f(\theta)$,

ii) $f$ is differentiable on $\theta$ and $-\theta$.

Then we have

$$
\lim _{n \rightarrow+\infty} S_{n}^{f}(\theta)=f(\theta) .
$$

Proof.

Let $n \in \mathbb{N}$ and $\theta \in\left[-\frac{\pi}{2}, \frac{\pi}{2}\right] \backslash\{0\}$. By Proposition 5.3, we can write

$$
f(\theta)-S_{n}^{f}(\theta)=\int_{-\frac{\pi}{2}}^{\frac{\pi}{2}}[f(\theta)-f(\phi)] D_{n}^{(\alpha, \beta)}(\theta, \phi) A_{\alpha, \beta}(\phi) d \phi
$$


From [4, Theorem 3.1], we have for all $\theta \neq \pm \phi$

$$
\begin{aligned}
f(\theta)-S_{n}^{f}(\theta) & =l_{n}^{(\alpha, \beta)} \int_{-\frac{\pi}{2}}^{\frac{\pi}{2}} \frac{f(\theta)-f(\phi)}{\cos (2 \theta)-\cos (2 \phi)}\left[\varphi_{n+1}^{(\alpha, \beta)}(\theta) \varphi_{n}^{(\alpha, \beta)}(\phi)-\varphi_{n}^{(\alpha, \beta)}(\theta) \varphi_{n+1}^{(\alpha, \beta)}(\phi)\right. \\
& +\frac{\lambda_{n}^{(\alpha, \beta)} \lambda_{n+1}^{(\alpha, \beta)}}{4(n+1)(n+\rho)} \\
& \left.\times\left(\Im \psi_{n+1}^{(\alpha, \beta)}(\theta) \Im \psi_{n}^{(\alpha, \beta)}(\phi)-\Im \psi_{n}^{(\alpha, \beta)}(\theta) \Im \psi_{n+1}^{(\alpha, \beta)}(\phi)\right)\right] A_{\alpha, \beta}(\phi) d \phi,
\end{aligned}
$$

where

$$
l_{n}^{(\alpha, \beta)}:=\frac{\Gamma(\alpha+n+2) \Gamma(\rho+n+1)}{2^{2 \rho-1}(\Gamma(\alpha+1))^{2}(2 n+\rho+1) n ! \Gamma(\beta+n+1)} .
$$

For all $\phi \in\left[-\frac{\pi}{2}, \frac{\pi}{2}\right] \backslash\{ \pm \theta\}$, we put

$$
g_{\theta}(\phi):=\frac{f(\theta)-f(\phi)}{\cos (2 \theta)-\cos (2 \phi)}
$$

Since we have supposed that $f$ is a piecewise continuous function on $\left[-\frac{\pi}{2}, \frac{\pi}{2}\right]$, then $g_{\theta}$ is also piecewise continuous on $\left[-\frac{\pi}{2}, \frac{\pi}{2}\right] \backslash\{ \pm \theta\}$.

Furthermore, we have

$$
\lim _{\phi \rightarrow \theta} g_{\theta}(\phi)=-\frac{1}{2} \frac{1}{\sin (2 \theta)} f^{\prime}(\theta)
$$

And from hypothese i) of our theorem, we deduce that

$$
\lim _{\phi \rightarrow-\theta} g_{\theta}(\phi)=\frac{1}{2} \frac{1}{\sin (2 \theta)} f^{\prime}(-\theta) .
$$

Under the assumption ii) of the theorem, these limits exist and are finite.

We still call $g_{\theta}$ the extension of $g_{\theta}$ on $\left[-\frac{\pi}{2}, \frac{\pi}{2}\right]$. Thus, $g_{\theta} \in L_{\alpha, \beta}^{2}$.

In the following, we denote by

$$
\begin{aligned}
\stackrel{\vee}{g}_{\theta}(\phi) & :=g_{\theta}(-\phi), \quad \phi \in\left[-\frac{\pi}{2}, \frac{\pi}{2}\right], \\
g_{\theta}^{1} & :=\left(g_{\theta}\right)_{\mid\left[0, \frac{\pi}{2}\right]}, \\
g_{\theta}^{2} & :=\left(g_{\theta}\right)_{\mid\left[-\frac{\pi}{2}, 0\right]}, \\
\stackrel{\vee}{g_{\theta}^{2}}(\phi) & :=g_{\theta}^{2}(-\phi), \quad \phi \in\left[0, \frac{\pi}{2}\right] .
\end{aligned}
$$

Now, we write

$$
f(\theta)-S_{n}^{f}(\theta)=I_{1}+I_{2}+I_{3}+I_{4}
$$


where

$$
\begin{aligned}
I_{1} & :=l_{n}^{(\alpha, \beta)} \varphi_{n+1}^{(\alpha, \beta)}(\theta) \int_{-\frac{\pi}{2}}^{\frac{\pi}{2}} g_{\theta}(\phi) \varphi_{n}^{(\alpha, \beta)}(\phi) A_{\alpha, \beta}(\phi) d \phi \\
I_{2} & :=-l_{n}^{(\alpha, \beta)} \varphi_{n}^{(\alpha, \beta)}(\theta) \int_{-\frac{\pi}{2}}^{\frac{\pi}{2}} g_{\theta}(\phi) \varphi_{n+1}^{(\alpha, \beta)}(\phi) A_{\alpha, \beta}(\phi) d \phi, \\
I_{3} & :=l_{n}^{(\alpha, \beta)} \frac{\lambda_{n}^{(\alpha, \beta)} \lambda_{n+1}^{(\alpha, \beta)}}{4(n+1)(n+\rho)} \Im \psi_{n+1}^{(\alpha, \beta)}(\theta) \int_{-\frac{\pi}{2}}^{\frac{\pi}{2}} g_{\theta}(\phi) \Im \psi_{n}^{(\alpha, \beta)}(\phi) A_{\alpha, \beta}(\phi) d \phi, \\
I_{4} & :=-l_{n}^{(\alpha, \beta)} \frac{\lambda_{n}^{(\alpha, \beta)} \lambda_{n+1}^{(\alpha, \beta)}}{4(n+1)(n+\rho)} \Im \psi_{n}^{(\alpha, \beta)}(\theta) \int_{-\frac{\pi}{2}}^{\frac{\pi}{2}} g_{\theta}(\phi) \Im \psi_{n+1}^{(\alpha, \beta)}(\phi) A_{\alpha, \beta}(\phi) d \phi .
\end{aligned}
$$

Combining the fact that

$$
l_{n}^{(\alpha, \beta)} \underset{+\infty}{\sim} \frac{1}{2^{2 \rho}(\Gamma(\alpha+1))^{2}} n^{2 \alpha+1},
$$

and the result (35) of [3], we get

$$
l_{n}^{(\alpha, \beta)} \varphi_{n+1}^{(\alpha, \beta)}(\theta) \underset{+\infty}{\sim} n^{\alpha+\frac{1}{2}} \frac{\cos \left[(2 n+2+\rho)|\theta|-(2 \alpha+1) \frac{\pi}{4}\right]}{\sqrt{\pi} \Gamma(\alpha+1) A_{\frac{2 \alpha-1}{4}, \frac{2 \beta-1}{4}}(\theta)} .
$$

Moreover, we have

$$
\int_{-\frac{\pi}{2}}^{\frac{\pi}{2}} g_{\theta}(\phi) \varphi_{n}^{(\alpha, \beta)}(\phi) A_{\alpha, \beta}(\phi) d \phi=\mathcal{F}_{\alpha, \beta}\left(g_{\theta}^{1}+\stackrel{\vee}{g_{\theta}^{2}}\right)(n) .
$$

From the Parseval formula for the Jacobi coefficients (see [2]), we obtain

$$
\mathcal{F}_{\alpha, \beta}\left(g_{\theta}^{1}+\stackrel{\vee}{g_{\theta}^{2}}\right)(n)=o\left(n^{-\left(\alpha+\frac{1}{2}\right)}\right) \text {. }
$$

Thus, $\lim _{n \rightarrow+\infty} I_{1}=0$.

We use the same proof as for $I_{1}$ to show that

$$
\begin{aligned}
& \lim _{n \rightarrow+\infty} I_{2}=\lim _{n \rightarrow+\infty}-\left[n^{\alpha+\frac{1}{2}} \frac{\cos \left[(2 n+\rho)|\theta|-(2 \alpha+1) \frac{\pi}{4}\right]}{\sqrt{\pi} \Gamma(\alpha+1) A_{\frac{2 \alpha-1}{4}, \frac{2 \beta-1}{4}}(\theta)} \mathcal{F}_{\alpha, \beta}\left(g_{\theta}^{1}+\stackrel{\vee}{g_{\theta}^{2}}\right)(n+1)\right] \\
& =0 \text {. }
\end{aligned}
$$

Otherwise, we have

$$
\int_{-\frac{\pi}{2}}^{\frac{\pi}{2}} g_{\theta}(\phi) \Im \psi_{n}^{(\alpha, \beta)}(\phi) A_{\alpha, \beta}(\phi) d \phi=\frac{1}{2 i} \mathcal{F}\left(\stackrel{\vee}{g_{\theta}}-g_{\theta}\right)(n) .
$$

By [2, corollary 3.5], we have

$$
\mathcal{F}\left(\stackrel{\vee}{g_{\theta}}-g_{\theta}\right)(n)=o\left(n^{-\left(\alpha+\frac{1}{2}\right)}\right) .
$$


Furthermore, we get, from [3, Theorem 4.7], that

$$
\Im \psi_{n+1}^{(\alpha, \beta)}(|\theta|) \underset{+\infty}{\sim} \frac{2^{2 \rho} \Gamma(\alpha+1)}{\sqrt{\pi}} \frac{n^{-\left(\alpha+\frac{1}{2}\right)}}{A_{\frac{2 \alpha-1}{4}, \frac{2 \beta-1}{4}}(\theta)} \sin \left[(2 n+2+\rho)|\theta|-(2 \alpha+1) \frac{\pi}{4}\right] .
$$

Since we have

$$
\lim _{n \rightarrow+\infty} \frac{\lambda_{n}^{(\alpha, \beta)} \lambda_{n+1}^{(\alpha, \beta)}}{4(n+1)(n+\rho)}=1
$$

then, we get

$$
\lim _{n \rightarrow+\infty} I_{3}=0 .
$$

We use the same reasons as for $I_{3}$ to show that

$$
\begin{aligned}
\lim _{n \rightarrow+\infty} I_{4} & =\lim _{n \rightarrow+\infty}-l_{n}^{(\alpha, \beta)} \frac{\lambda_{n}^{(\alpha, \beta)} \lambda_{n+1}^{(\alpha, \beta)}}{4(n+1)(n+\rho)} \Im \psi_{n}^{(\alpha, \beta)}(\theta) \int_{-\frac{\pi}{2}}^{\frac{\pi}{2}} g_{\theta}(\phi) \Im \psi_{n+1}^{(\alpha, \beta)}(\phi) A_{\alpha, \beta}(\phi) d \phi \\
& =0 .
\end{aligned}
$$

Hence, we obtain

$$
\lim _{n \rightarrow+\infty}\left[f(\theta)-S_{n}^{f}(\theta)\right]=\lim _{n \rightarrow+\infty} I_{1}+I_{2}+I_{3}+I_{4}=0 .
$$

Which achieves the proof.

\section{REFERENCES}

[1] H. Bavinck, Approximation processes for Fourier-Jacobi expansions, Appl. Anal., 5 (1976), 293312 .

[2] F. Chouchene, Harmonic analysis associated with the Jacobi-Dunkl operator on $]-\frac{\pi}{2}, \frac{\pi}{2}[$, J. Comput. Appl. Math. 178 (2005), 75-89.

[3] F. Chouchene, Bounds, asymptotic behavior and recurrence relations for the Jacobi-Dunkl polynomials, Int. J. Open Problems Complex Analysis, 6 (1)(2014), 49-77.

[4] F. Chouchene, Recurrence and Christoffel-Darboux formulas for the Jacobi-Dunkl polynomials and applications, Comm. Math. Anal., 16 (1)(2014), 123-142.

[5] G.B. Folland, Fourier analysis and its applications, Pacific Grove, CA: Wadsworth \& Brooks/Cole Advanced Books \& Software, 1992.

[6] J.-B. J. Fourier, Mémoire sur la propagation de la chaleur dans les corps solides, (nepublikováno) pro Institute de France, Paris, podno 21. prosince, 1807.

[7] J.-B. J. Fourier, Théorie analytique de la chaleur. Didot, Paris, 1822.

[8] G. Gasper, Positivity and the convolution structure for Jacobi series, Ann. Math. 93 (1971), 112-118.

[9] L. Grafakos, Classical Fourier Analysis, $3^{d}$ edition, Springer, New York, 2014.

[10] J.-P. Kahane, P.G. Lemarié-Rieusset, Séries de Fourier et Ondelettes, Cassini, 1998.

[11] G. Kavernadze, Uniform convergence of Fourier-Jacobi series, J. Approx. Theory, 117 (2002), 207-228.

[12] A. Lesfari, Distributions, Analyse de Fourier et transformation de Laplace, Ellipses dition Marketing, 2012.

[13] Z. Li, Y. Xu, Summability of the product Jacobi series, J. Approx. Theory, 104 (2000), 287-301.

[14] H.N. Hhaskar, S. Tikhonov, Wiener type theorems for Jacobi series with nonnegative coefficients, Proc. Amer. Math. Soc., 140 (3)(2012), 977-986.

[15] G. Szego, Orthogonal polynomials, Amer. Math. Soc. Colloq. Pub. Vol. 23, Amer. Math. Soc. Providence, R. I., 1975. 
[16] Y. Xu, Mean convergence of generalized Jacobi series and interpolating polynomials, I, J. Approx. Theory, 72 (1993), 237-251.

[17] Y. Xu, Mean convergence of generalized Jacobi series and interpolating polynomials, II, J. Approx. Theory, 76 (1994), 77-92.

[18] W. zu Castell, F. Filbir, and Y. Xu, Cesàro means of Jacobi expansions on the parabolic biangle, J. Approx. Theory 159 (2009), 167-179.

E-mail address: frej.chouchene@essths.u-sousse.tn

E-mail address: iness.haouala@yahoo.fr 